\title{
Effects of binary mixtures of estrogen and antiestrogens on Japanese medaka (Oryzias latipes)
}

\author{
Liwei Sun $^{\mathrm{a}, \mathrm{b}}$, Jinmiao Zha ${ }^{\mathrm{b}}$, Zijian Wang ${ }^{\mathrm{b}, *}$ \\ a College of Biological and Environmental Engineering, Zhejiang University of Technology, No. 18 Chaowang Road, Hangzhou, 310032, PR China \\ b State Key Laboratory of Environmental Aquatic Chemistry, Research Center for Eco-Environmental Sciences, Chinese Academy of Sciences, P.O. Box 2871, \\ Shuangqing Rd 18, Haidian District, Beijing, 100085, PR China
}

\section{A R T I C L E I N F O}

\section{Article history:}

Received 16 October 2008

Received in revised form 7 March 2009

Accepted 31 March 2009

\section{Keywords:}

Mixture

Endocrine-disrupting chemical

Japanese medaka (Oryzias latipes)

Reproduction

\begin{abstract}
A B S T R A C T
It is supposed that the estrogenic effects of estrogenic chemicals will be balanced by the presence of other endocrine-disrupting chemicals (EDCs) with different properties. However, little convincing research into this issue has been carried out to date. In the present study, the combined effects of EDCs with estrogenic and antiestrogenic activities were assessed using a 21-day reproduction assay with the Japanese medaka (Oryzias latipes) as an in vivo model. Natural estrogen (17 $\beta$-estradiol, E2) and the pharmaceutical substances letrozole and tamoxifen, which are both model antiestrogens, were tested as reference compounds individually and in combination. Exposure to E2 (200 ng/L) alone resulted in significant biological changes in paired fish, including impaired reproductive capacity and plasma vitellogenin induction. Upon coexposure with tamoxifen or letrozole $(10,50$ and $250 \mu \mathrm{g} / \mathrm{L})$, the effects of estrogen on some biomarkers (e.g., plasma vitellogenin concentration) could be neutralized to some extent, but the impairments in reproductive performance were hardly ameliorated or even became more severe. Since reproductive performance is a more holistic parameter with population-level relevance, the possibility that the effects of estrogenic chemicals can be cancelled out by the presence of antiestrogens in aquatic organisms should be questioned. Our observations also illustrate the importance of considering multiple endpoints at different biological levels of organisms for accurate and adequate assessment of the integrated effects of EDCs, especially in environmentally relevant exposure situations.
\end{abstract}

(c) 2009 Elsevier B.V. All rights reserved.

\section{Introduction}

A considerable amount of attention has been paid to the so-called environmental endocrine-disrupting chemicals (EDCs), which constitute a broad group of environmental pollutants that are able to mimic or antagonize the effects of endogenous hormones, or to disrupt the synthesis and metabolism of endogenous hormones and hormone receptors (U.S.EPA, 2002; Rodriguez-Mozaz et al., 2004). Owing to the powerful weight of evidence that many water bodies receive significant inputs of natural and synthetic chemicals (including various EDCs), most reported cases of endocrine disruption and associated reproductive impairment in wildlife have involved aquatic organisms, particularly various species of fish (Tyler et al., 1998; Sheahan et al., 2002). The significant effects

Abbreviations: DWC, dilution water control; E2, 17 $\beta$-estradiol; EDC, endocrinedisrupting chemical; ER, estrogen receptor; GSI, gonadosomatic index; HSI, hepatosomatic index; LET, letrozole; SC, solvent control; TAM, tamoxifen; TBT, tributyltin; VTG, vitellogenin.

* Corresponding author. Tel.: +86 106284 9140; fax: +86 1062849140 .

E-mail address: wangzj@rcees.ac.cn (Z. Wang). observed in fish include reduced levels of reproductive hormones (i.e., estrogens and androgens), inhibited gonadal growth, the appearance of a female egg protein (vitellogenin; VTG) in male fish, gonadal histopathologies, and even intersex fish containing ovotestes (Jobling et al., 2002).

In reality, aquatic organisms are rarely exposed to single chemicals in isolation but rather to complex mixtures of chemicals (Thorpe et al., 2006). Therefore, combination effects require urgent attention when assessing the potential risks of EDCs. During the last decade, many articles on the combined effects of EDCs have been published and have made significant contributions to this field of research (Brian et al., 2007; Kortenkamp, 2007). However, the studies have mostly concentrated on assessing if chemicals that act via the same mechanism induce a common, strengthened effect (Brian et al., 2007), studying especially chemicals with the potential to act as estrogen mimics and evoke responses similar to $17 \beta$-estradiol (E2) (Brian et al., 2007; Filby et al., 2007; Kortenkamp, 2007). In contrast, comparatively few studies have been performed on mixtures of different classes of EDCs (Brian et al., 2007; Kortenkamp, 2007).

Overt endocrine disruption in fish does not appear to be a ubiquitous environmental phenomenon, but rather appears to be 
more frequently associated with higher levels of contamination or in some extreme areas predominantly contaminated with EDCs belonging to the same class, such as in the downstream of sewage treatment plants (Purdom et al., 1994; Thorpe et al., 2006). Thus it is supposed that the estrogenic effects of environmental chemicals will be balanced by the presence of other chemicals with different properties, such as antiestrogens and androgens, in real exposure situations (Witorsch, 2002). However, to the best of our knowledge, little convincing research into this issue has been carried out in either the field or the laboratory.

The main purpose of the present study was to evaluate the combined effects of EDCs with estrogenic and antiestrogenic activities using the Japanese medaka as an in vivo model. To achieve this, natural estrogen (E2) and two model antiestrogens, letrozole (LET) and tamoxifen (TAM), were selected as representative chemicals. In general, antiestrogens can be classified into two broad groups based on their mechanism of action. Pollutants that interfere with estrogen signaling through competitive inhibition of estrogen binding to the estrogen receptor (ER) are considered to be direct-acting antiestrogens. On the contrary, contaminants that alter the normal turnover of estrogen in fish are considered to be indirect-acting antiestrogens, for which decreased synthesis of E2 through aromatase inhibition appears to have the most biological significance (U.S.EPA, 2002). TAM belongs to the direct-acting antiestrogens, and its pharmaceutical action is that of an ER antagonist that forms a relatively stable complex with the ER, thereby reducing the number of receptors available for subsequent E2 binding (Jordan et al., 1977). It is actually a weak ER agonist at low concentrations and an ER antagonist at high concentrations (U.S.EPA, 2002), and is widely used in the treatment of primary and recurrent breast cancer (Carlson, 1997). LET, with the commonly used brand name Femara, is one of the most potent aromatase inhibitors developed to date (Smith, 1999). It is capable of inhibiting aromatase by $98-99 \%$ and reducing the serum concentrations of estrone and E2 to below their limits of detection in patients (Smith, 1999), and has been approved as a first-line therapy for hormone receptor-positive metastatic breast cancer (Haynes et al., 2003).

The medaka has been recognized as an appropriate model for the evaluation of EDCs (Patyna et al., 1999). In particular, its large eggs with transparent chorion offer the advantages of continuous and non-disruptive observation of the developing embryos. In the present study, we attempted to obtain information regarding the nature of the chemical interactions to learn whether the presence of antiestrogens can cancel out the effects of estrogenic chemicals in aquatic organisms and if so, to what degree.

\section{Materials and methods}

\subsection{Test chemicals}

E2 and TAM (purity, >99\%) were purchased from Sigma Chemical Co. (USA). LET (purity, >98.0\%) was obtained from Beijing DezhongVenture Pharmaceutical Technology Development Co. Ltd. (China). Owing to the low solubility of these chemicals in water, stock solutions were prepared in HPLC-grade acetone (Fisher Scientific, USA). All other chemicals used were of analytical or HPLC grade.

\subsection{Test fish}

The Japanese medaka (d-rR) stock used in this study originated from the Laboratory of Freshwater Fish at the Bioscience Center of Nagoya University (Japan). The brood stock has been maintained and propagated in our laboratory for more than 5 years. The medaka was maintained in charcoal-dechlorinated tap water ( $\mathrm{pH} 7.2-7.6)$ at a constant temperature $\left(25 \pm 1^{\circ} \mathrm{C}\right)$ with a 16 -h light/8-h dark photoperiod. The brood stock was fed three times daily, once with brine shrimp (Artemia nauplii) and twice with commercial food $\left(\right.$ TetraMin $\left.^{\circledR}\right)$.

\subsection{Exposure conditions for medaka reproduction}

Breeding pairs of medaka (approximately 5 months of age) were acclimated for 3 weeks in flow-through 15-L glass aquaria under the same conditions described in Section 2.2. During the last week of acclimation, newly spawned eggs were collected daily within a few hours after oviposition, counted and assessed for fertility. Only the pairs whose fecundity and fertility met the criteria (fecundity $>15$ eggs/day for each female and fertility $>90 \%$ ) were selected for the reproductive test. Six pairs were randomly assigned to a $15-\mathrm{L}$ glass aquarium and duplicate aquaria were used for each exposure level. A dilution apparatus equipped with a peristaltic pump and mixing chambers supplied a constant flow equivalent to 7-fold the aquarium volume per day.

Based on the results of our previous studies (Sun et al., 2007a,b) and a separate study (Kang et al., 2002), the fish were exposed to a nominal $\mathrm{E} 2$ concentration of $200 \mathrm{ng} / \mathrm{L}$ as a positive treatment group, binary mixtures of E2 + LET at concentrations of $200 \mathrm{ng} / \mathrm{L}+10 \mu \mathrm{g} / \mathrm{L}$, $200 \mathrm{ng} / \mathrm{L}+50 \mu \mathrm{g} / \mathrm{L}$ and $200 \mathrm{ng} / \mathrm{L}+250 \mu \mathrm{g} / \mathrm{L}$ or binary mixtures of $\mathrm{E} 2+\mathrm{TAM}$ at $200 \mathrm{ng} / \mathrm{L}+10 \mu \mathrm{g} / \mathrm{L}, 200 \mathrm{ng} / \mathrm{L}+50 \mu \mathrm{g} / \mathrm{L}$ and $200 \mathrm{ng} / \mathrm{L}+250 \mu \mathrm{g} / \mathrm{L}$. The solvent control (SC) aquaria and all exposure aquaria received $0.1 \mathrm{~mL} / \mathrm{L}$ acetone, while the dilution water control (DWC) aquaria received dechlorinated tap water only.

The test duration was 21 days and the feeding regime was maintained throughout the exposure period. During the last week of exposure, newly spawned eggs were carefully collected within a few hours after fertilization. The number of eggs spawned in each treatment group was counted, and the ratio of fertilized eggs was calculated. Each embryo was maintained in dechlorinated tap water and observed daily. The hatchability, time to hatching and gross abnormality rate were recorded. Gross abnormalities were characterized according to Nimrod and Benson (1998), and one of the most common abnormalities was scoliosis. Once hatched, the larvae were maintained for 15 days and the genetic sex ratio was determined by the color of the fish. In the d-rR strain of medaka, sexlinked genes determine orange-red coloration in males and white coloration in females (Yamamoto, 1953). At the end of the F0 generation exposure, all the adult fish were sampled. Length and weight were measured, and a condition factor $K$ was calculated using the formula $K=($ weight $[\mathrm{g}] \times 100) /(\text { length }[\mathrm{cm}])^{3}$. The livers and gonads were sampled, and the gonadosomatic index (GSI; \%) and hepatosomatic index (HSI; \%) were calculated as the ratios of the gonad and liver weights to the body weight, respectively.

\subsection{Blood sampling and measurement of plasma VTG}

After each exposure, the fish were sacrificed by placing on ice for 1-2 min, and blood samples were collected into heparinized microcapillary tubes by severing the caudal peduncle. After measurement of the volume, sample buffer (20 mM Tris- $\mathrm{HCl}$ pH 7.5, 1 mM EDTA, $150 \mathrm{mM} \mathrm{NaCl}$ and $25 \mathrm{KIU} / \mathrm{mL}$ aprotinin) was added to each tube. The samples were immediately centrifuged $\left(8000 \times \mathrm{g}, 10 \mathrm{~min}, 4^{\circ} \mathrm{C}\right)$, and the obtained plasma samples were stored at $-80^{\circ} \mathrm{C}$ until analysis.

Plasma VTG levels were measured using a competitive enzymelinked immunosorbent assay as described previously (Brion et al., 2002). The working range of the assay was from 30 to $1500 \mathrm{ng} / \mathrm{mL}$ (20-80\% binding), and the minimal detection limit was $15 \mathrm{ng} / \mathrm{mL}$ for purified VTG. To avoid matrix effect, a dilution of at least 1:100 was chosen for the routine assay. The intra- and inter-assay variations at $50 \%$ binding were $5.6 \%$ and $10.2 \%$, respectively. Medaka VTG was separated and purified as previously described (Yamanaka et al., 
1998), and then used as a standard. VTG in diluted samples was measured in triplicate.

\subsection{Analytical chemistry}

The concentrations of chemicals were determined at least four times during the experiment. For measurement of E2, the analysis was performed according to the methods of Quintana et al. (2004). Briefly, filtered water samples were spiked with deuterated standards, and extracted using preconditioned solid-phase extraction (SPE) cartridges. E2 was eluted in methanol/tert-butyl methyl ether $(1 / 9 ; \mathrm{v} / \mathrm{v})$ and derivatized. The samples were analyzed using a 6890 gas chromatograph/5973 mass spectrometer (Agilent, USA). For measurement of LET, the analytical method was performed as described by Pfister et al. (1994) with some modifications (Sun et al., 2007b). The water samples were liquid/liquid extracted using dichloromethane. Extracts were analyzed on a high performance liquid chromatography system (Dionex, US) with an ultraviolet detector set as $234 \mathrm{~nm}$. The $\mathrm{C}_{18}$ column was used, and the mobile phase was $70 \%$ phosphate buffer $(0.01 \mathrm{M}$; pH 7.0) and $30 \%$ acetonitrile $(\mathrm{v} / \mathrm{v})$. For the measurement of TAM, the analytical method was adapted from Mihailescu et al. (2000). The water samples were extracted using SPE and eluted with methanol. Eluents were evaporated to dryness and derivatized. The GC/MS system was used for the determination of TAM concentrations.

The analyzed concentration (mean \pm standard deviation, and \% analyzed/nominal) of E2 was $187.2 \pm 15.1 \mathrm{ng} / \mathrm{L}$ (93.6\%). For LET, the measured concentrations were $9.3 \pm 0.9 \mu \mathrm{g} / \mathrm{L}$ (93.0\%), $45.8 \pm 6.6 \mu \mathrm{g} / \mathrm{L}$ (91.6\%) and $243.5 \pm 10.1 \mu \mathrm{g} / \mathrm{L}$ (97.4\%). For TAM, the measured concentrations were $8.8 \pm 1.6 \mu \mathrm{g} / \mathrm{L}(88.0 \%)$, $45.1 \pm 1.6 \mu \mathrm{g} / \mathrm{L}(90.2 \%)$ and $232.1 \pm 16.3 \mu \mathrm{g} / \mathrm{L}$ (92.8\%). No analyte was detected in SC or DWC. Since good agreement existed between the nominal and measured exposure concentrations, nominal values were used in the following text.

\subsection{Statistical analysis}

Prior to data analysis, $\chi^{2}$ analyses, Student's t-tests (parametric data) or Mann-Whitney rank sum tests (nonparametric data, nonequal variances) were used to test for differences between the DWC and SC groups. The values in the two control groups did not differ in any of the cases and were therefore pooled for subsequent analyses. The experimental data were checked for assumptions of normality and homogeneity of variance using the Kolmogorov-Smirnov one-sample test and Levene's test, respectively. When the assumptions were met, the data were subjected to one-way analysis of variance (ANOVA) with comparisons of specific means by Dunnett's test. When the data failed either test, the nonparametric Kruskal-Wallis test was used. When VTG was not detected in male fish, we used the detection limit as the VTG value. To avoid biases associated with size-specific indices (Packard and Boardman, 1999; Kang et al., 2002), analysis of covariance (ANCOVA) with the body weight of the medaka as a covariate was used to compare the GSI and HSI values among all experimental groups. The data pertaining to sex ratios were assessed by $\chi^{2}$ analysis. The level for statistical significance was set at $p \leq 0.05$. All statistical analyses were carried out using SPSS 13.0 (SPSS, Chicago, IL, USA) and Origin 7.0 (OriginLab, Northampton, MA, USA).

\section{Results}

\subsection{Organism parameters in adult medaka}

No mortalities were observed among fish exposed to any of the test chemicals in any of the experiments, indicating that the chemicals tested were not acutely toxic at the concentrations used and the fish were not unduly stressed. Moreover, exposure to E2 alone or the binary mixtures did not affect the body length, weight or condition factor $K$ of either sex (data not shown).

The HSI and GSI values in adult male and female medaka after 21 days of exposure are shown in Fig. 1. ANCOVA revealed no differences in the GSI values of female medaka between the control group and any of the treatment groups. For the males, exposure to E2 alone or combination with LET caused no discernable changes $(p>0.05)$ in the GSI. In contrast, the GSI value increased for exposure to binary mixtures of E2 and TAM. Significant increases $(p<0.05)$ were found for coexposure to E2 and TAM at 50 or $250 \mu \mathrm{g} / \mathrm{L}$ compared with the control group, while all the GSI values were significantly increased in fish exposed to binary mixtures of E2 and TAM relative to exposure to E2 alone $(p<0.05)$.

For males, exposure to E2 resulted in a significant increase $(p<0.05)$ in the HSI compared with the control group. Compared with the E2 treatment group, coexposure to E2 and LET at 10 or $50 \mu \mathrm{g} / \mathrm{L}$ caused no change in the HSI, while a lower HSI value was observed in males coexposed to E2 and LET at $250 \mu \mathrm{g} / \mathrm{L}(p<0.05)$. All the coexposure treatments with E2 and TAM caused reductions in the HSI compared with E2 treatment alone $(p<0.05)$, while no significant differences were observed in male medaka coexposed to E2 and TAM at 50 or $250 \mu \mathrm{g} / \mathrm{L}$ relative to the control group. With respect to the females, exposure to E2 caused a significant increase $(p<0.05)$ in the HSI compared with the control group, while coexposure to E2 and LET caused concentration-dependent decreases in the HSI, and no significant differences were found relative to the control group for all three coexposure groups. For TAM, coexposure resulted in a significant decrease $(p<0.05)$ in the HSI compared with exposure to E2 alone, except in the highest concentration group $(250 \mu \mathrm{g} / \mathrm{L})$.

\subsection{Plasma VTG in adult medaka}

No VTG was detected in the plasma of control adult male medaka (Fig. 2). Exposure to E2 (200 ng/L) alone induced a significantly elevated level of VTG synthesis with a mean concentration exceeding $4.5 \mathrm{mg} / \mathrm{mL}$, while coexposure to E2 and LET did not affect the VTG concentration $(p>0.05)$. In the case of coexposure to E2 and TAM, the VTG concentration decreased in a TAM concentrationdependent way, and significant differences $(p<0.05)$ were observed for the 50 and $250 \mu \mathrm{g} / \mathrm{L}$ TAM treatments.

In females, the plasma VTG concentration was increased by E2, but no significant difference was observed compared with the control group (Fig. 2). All coexposure treatments with E2 and LET significantly inhibited VTG synthesis $(p<0.05)$ compared with either the control or E2 treatment groups. Similar effects were observed in females exposed to the mixtures of E2 and TAM.

\subsection{Reproductive effects}

Compared with the control group, both the fecundity and fertility of the medaka decreased significantly $(p<0.05)$ after exposure to E2 (200 ng/L) (Fig. 3). After combined exposure to LET, the effects of E2 remained unchanged or became even more severe at the highest concentration $(250 \mu \mathrm{g} / \mathrm{L})$ and appeared to be concentration-dependent. Similar effects were found for the mixtures of E2 and TAM. In the highest exposure group (200 ng/L $\mathrm{E} 2+250 \mu \mathrm{g} / \mathrm{L} \mathrm{TAM})$, the medaka ceased spawning during the final week of exposure.

The hatchabilities, times to hatching, gross abnormality rates and genetic sex ratios of the F1 generation are shown in Table 1 , excluding the mixture of E2 $(200 \mathrm{ng} / \mathrm{L})$ and TAM $(250 \mu \mathrm{g} / \mathrm{L})$ for which no embryos were collected. Paired medaka (F0 generation) exposed to E2 alone tended to exhibit a slight reduction in the hatchability of fertilized eggs (F1 generation), but no significant 

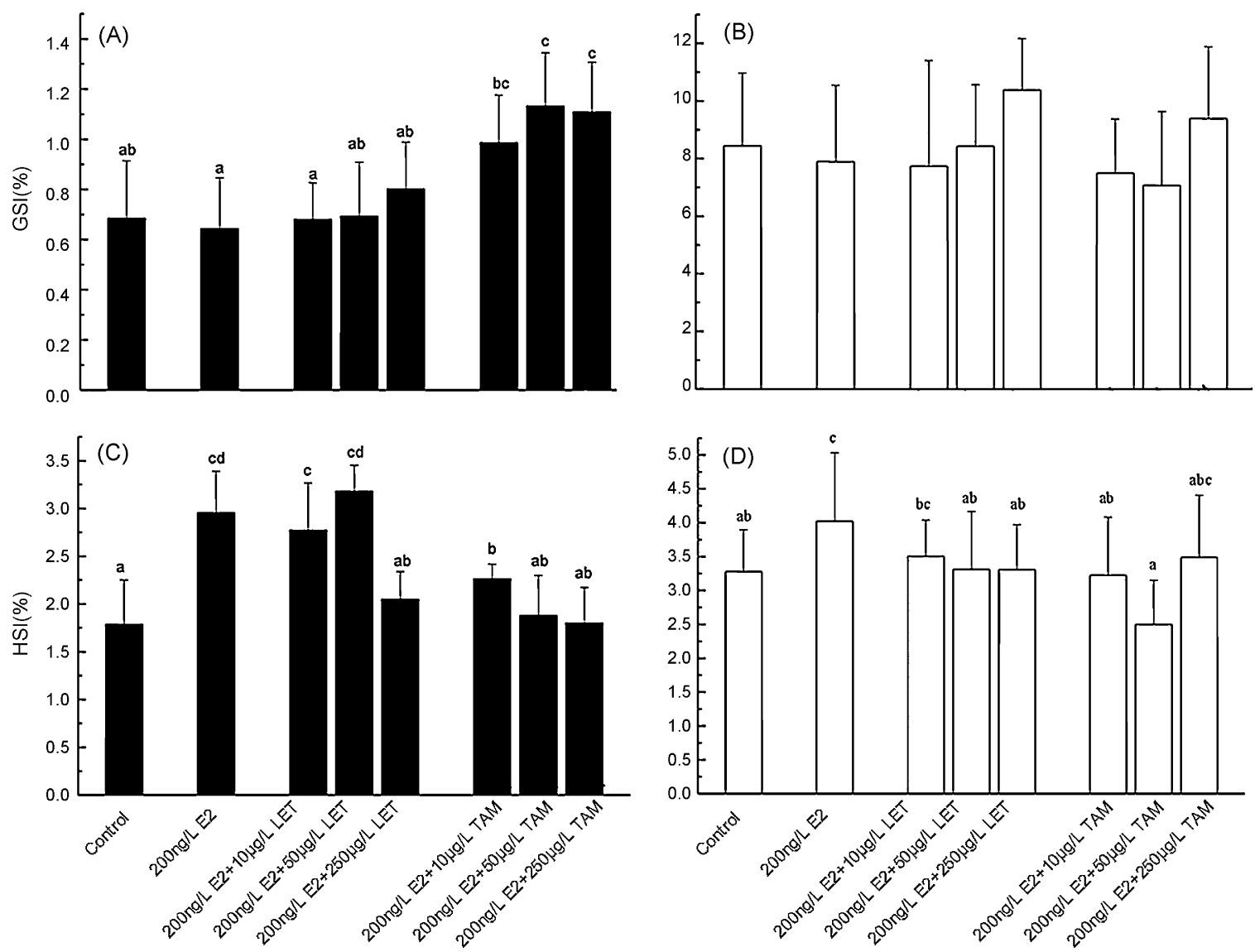

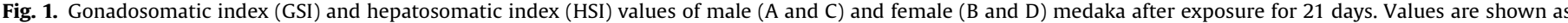
means + S.D. Significant differences between experimental groups are denoted by different letters $(p<0.05)$.

difference was found compared with the control group. No changes were observed in the hatchability after coexposure to E2 and LET. In contrast, the hatchability was significantly decreased $(p<0.05)$ after coexposure to E2 and TAM $(50 \mu \mathrm{g} / \mathrm{L})$. Similar trends were observed in the gross abnormality rate, i.e., only coexposure to E2 and TAM $(50 \mu \mathrm{g} / \mathrm{L})$ caused a significant increase relative to the control group. The time to hatching was also significantly increased $(p<0.05)$ for

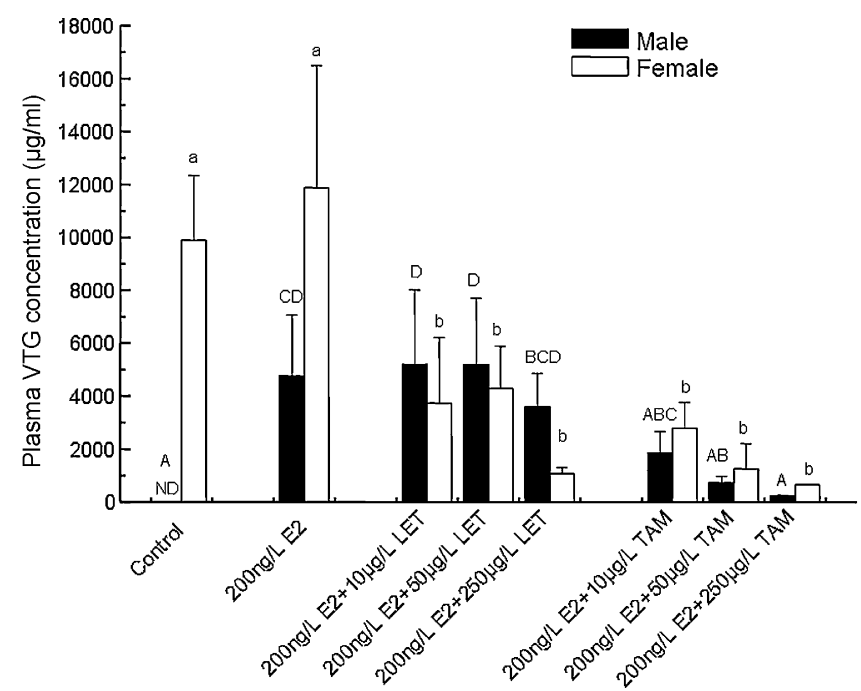

Fig. 2. Plasma vitellogenin (VTG) concentrations of medaka after exposure for 21 days. Values are shown as means + S.D. Significant differences between experimental groups are denoted by different letters $(p<0.05)$.

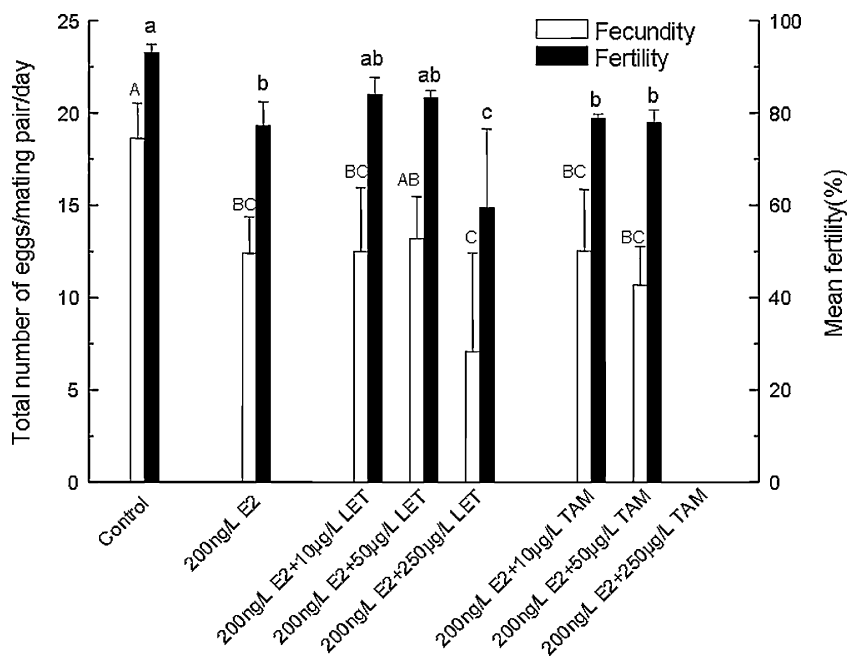

Fig. 3. Fecundity and fertility of eggs from paired mature medaka in the last week of a 21-day exposure period. Values are shown as means + S.D. Significant differences between experimental groups are denoted by different letters $(p<0.05)$.

embryos coexposed to E2 and TAM $(50 \mu \mathrm{g} / \mathrm{L})$ relative to the control group, although no difference was detected compared with exposure to E2 alone. When adult medaka (F0) was exposed to E2 alone, the genetic sex ratio of the juvenile medaka (F1) was not affected. However, exposure to E2 combined with high concentrations of LET $(250 \mu \mathrm{g} / \mathrm{L})$ and TAM $(50 \mu \mathrm{g} / \mathrm{L})$ resulted in significant shifts of the sex ratios in the juvenile population, with increased ratios of males to females. 
Table 1

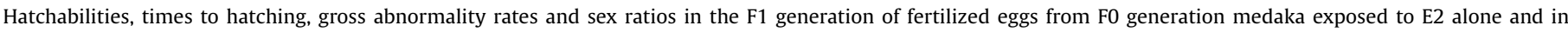
combination with LET or TAM for 21 days $^{\mathrm{a}}$.

\begin{tabular}{|c|c|c|c|c|}
\hline Treatment (/L) & Hatchability (\%) & Time to hatching (day) & Gross abnormality rate (\%) & Sex ratio $\left(\%, \sigma^{7}: q\right)$ \\
\hline Control & $90.7 \pm 3.3 \mathrm{~A}$ & $8.16 \pm 0.16 \mathrm{~A}$ & $1.0 \pm 1.6 \mathrm{~A}$ & $55: 45$ \\
\hline E2: $200 \mathrm{ng}$ & $74.9 \pm 15.2 \mathrm{~A}$ & $8.57 \pm 0.37 \mathrm{AB}$ & $2.0 \pm 3.4 \mathrm{~A}$ & $52: 48$ \\
\hline E2: $200 \mathrm{ng}+\mathrm{LET}: 10 \mu \mathrm{g}$ & $79.7 \pm 7.9 \mathrm{~A}$ & $8.35 \pm 0.12 \mathrm{AB}$ & $7.7 \pm 4.3 \mathrm{~A}$ & $60: 40$ \\
\hline E2: $200 \mathrm{ng}+\mathrm{LET}: 50 \mu \mathrm{g}$ & $85.7 \pm 4.9 \mathrm{~A}$ & $8.70 \pm 0.15 \mathrm{AB}$ & $1.0 \pm 1.7 \mathrm{~A}$ & $47: 53$ \\
\hline E2: 200 ng + LET: $250 \mu \mathrm{g}$ & $76.5 \pm 10.6 \mathrm{~A}$ & $8.32 \pm 0.33 \mathrm{AB}$ & $1.4 \pm 2.5 \mathrm{~A}$ & $71: 29^{*}$ \\
\hline E2: $200 \mathrm{ng}+\mathrm{TAM}: 10 \mu \mathrm{g}$ & $88.1 \pm 5.9 \mathrm{~A}$ & $8.80 \pm 0.54 \mathrm{AB}$ & $3.8 \pm 6.6 \mathrm{~A}$ & $52: 48$ \\
\hline E2: $200 \mathrm{ng}+\mathrm{TAM}: 50 \mu \mathrm{g}$ & $43.1 \pm 26.9 \mathrm{~B}$ & $9.07 \pm 0.59 \mathrm{~B}$ & $42.2 \pm 23.4 \mathrm{~B}$ & $100: 0^{* * *}$ \\
\hline E2: $200 \mathrm{ng}+\mathrm{TAM}: 250 \mu \mathrm{g}$ & & & & \\
\hline
\end{tabular}

a Data are shown as means \pm S.D. Significant differences between experimental groups are denoted by different letters $(p<0.05)$. For sex ratio analyses, the $\chi^{2}$ test was

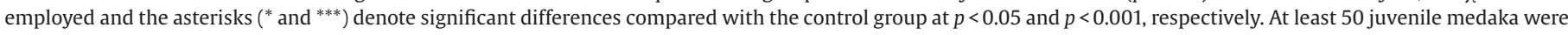

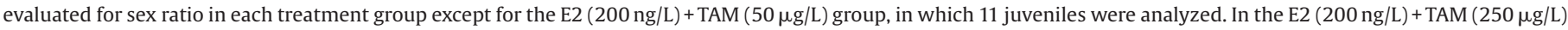
group, F0 generation medaka ceased spawning during the final week of exposure, therefore no analyses was performed.

\section{Discussion}

Exposure to E2 has been reported to cause both subtle and profound changes in the growth and morphology in fish, especially in males. The results of the present study revealed that plasma VTG was sharply induced and the HSI was significantly increased in male medaka exposed to E2 ( $200 \mathrm{ng} / \mathrm{L}$ ). In contrast, exposure to E2 led to slight effects in female medaka, probably owing to the relatively higher levels of endogenous E2 in the females compared to the males. However, with respect to paired medaka, the reproductive performance, involving both fecundity and fertility, was significantly impaired. These observations are consistent with those in previous studies (e.g., Kang et al., 2002).

When combined with LET, the adverse effects of E2 on reproductive performance could not be neutralized. These findings are consistent with the known mechanism of LET, which inhibits aromatase and reduces the synthesis of endogenous estrogen, and exposure to LET resulted in more pronounced adverse effects on females than on males. The adverse effects of E2 on males could not be ameliorated by coexposure with LET, which was substantially reflected in the reproductive performance. In our previous study (Sun et al., 2007b), we showed that exposure to LET alone reduced plasma VTG levels in females in a dose-dependent manner. Moreover, dose-dependent decreases in fecundity and fertility accompanied by histological changes in females (inhibition of oocyte growth and possibly maturation) were observed. For males, no significant changes were found except for changes in histology in fish exposed to high levels of LET (Sun et al., 2007b).

On the other hand, when combined with TAM exposure, the plasma VTG concentrations induced by E2 were significantly reduced in both sexes, and were even lower than those in the control group for females. Since VTG synthesis is initially triggered by binding of estrogen or an estrogen mimic to the ER, the reduction in VTG levels can be attributed to the properties of TAM as a competitive inhibitor of E2. The inhibition of VTG synthesis also explains the observed reduction in the HSI because the weight of the liver will increase owing to VTG accumulation. However, coexposure to TAM could not neutralize the impaired reproductive capacity caused by E2, and complete reproductive failure occurred at the highest concentration $(250 \mu \mathrm{g} / \mathrm{L})$. In our previous study (Sun et al., 2007a), exposure to TAM alone affected the reproductive capacity detrimentally at a concentration of only $625 \mu \mathrm{g} / \mathrm{L}$, but no paired medaka ceased spawning. Therefore, these findings demonstrate that combined exposure to E2 and TAM can impair the reproductive function more severely than exposure to each component alone.

When adult medakas were exposed to E2 alone, no significant transgenerational effects were observed in their progeny. Therefore, the detrimental changes in hatchability, time to hatching, gross abnormality rate and genetic sex ratio can mostly be ascribed to LET or TAM exposure. Sex inversions in fish exposed to EDCs have been reported in previous studies. However, to the best of our knowledge, the phenomenon of skewed genetic sex ratios observed in the present study, as well as the one we reported previously (Sun et al., 2007a,b), has not been described by other researchers. We hypothesized that the observed changes may be caused by differential sensitivities of spermatozoa to the tested chemicals (i.e., spermatozoa with the $\mathrm{X}$ chromosome may be more sensitive than spermatozoa with the $Y$ chromosome), resulting in more spermatozoa with the Y chromosome. In fact, it has been reported that exposure to the toxicant bisazir can lead to DNA damage in sea lamprey (Petromyzon marinus) spermatozoa (Ciereszko et al., 2005). Unfortunately, we did not have any methods for distinguishing between spermatozoa with different sex chromosomes and were therefore unable to confirm this hypothesis.

Previous studies have shown that coexposure to EDCs with different modes of action can result in very different effects compared with those of exposure to individual EDCs. Coexposure assays can be helpful in clarifying the specific patterns of EDCs, particularly in defining the toxic modes of action, but more importantly, they can serve as a reliable approach for assessing the combined effects of EDCs that belong to different categories under real exposure situations. Kawahara and Yamashita (2000) demonstrated that coexposure with TAM blocked the shift in phenotype from males to females caused by E2 exposure using juvenile medaka in vivo. Similarly, Elias et al. (2007) reported that the presence of TAM in the environment appears to be able to mask many signs of estrogen exposure, including the changes in secondary sexual characteristics, hypertrophy of ovaries and testes, and fatty infiltration of organs in rainbow darters (Etheostoma caeruleum). A study with another EDC, tributyltin (TBT), indicated that $17 \alpha$-ethinylestradiol at environmentally relevant concentrations can block the masculinizing effects of TBT on zebrafish (Danio rerio), suggesting that the presence of estrogens in the aquatic environment may neutralize the masculinizing effects of TBT on fish (Santos et al., 2006). Although the mechanism for the TBT-induced masculinization of fish is still not fully understood, some lines of evidence suggest the involvement of P450 aromatase or the suppression of its gene expression, which is very similar to the mechanism of LET. However, the results of the present study have demonstrated that, although antiestrogens can neutralize the effects of estrogen to some extent on some biomarkers (e.g., VTG), the impairment in reproductive performance was hardly ameliorated, and in some cases became even more severe. In addition, as mentioned earlier, these two human pharmaceutical compounds were chosen as references for estrogen antagonist activity. However, even if the initial signal transduction mechanisms are similar, the physiological outcomes in teleost fish may differ markedly from the primary therapeutic pharmacology observed in humans. It is possible that some chemicals may be antiestrogenic toward the liver, leading to VTG reduction, but act via different mechanisms through recep- 
tors or enzyme systems to exert effects at other sites. Therefore, our present observations coupled with those in previous studies (Van der Ven et al., 2007; Martinovi et al., 2008) suggest the importance of assessing multiple endpoints at different biological levels of organisms, rather than a few commonly used biomarkers, when evaluating the effects of possible EDCs or their combinations.

Biomarkers have become important tools in the assessment of EDCs in aquatic life. However, the extent to which biomarkers are able to provide unambiguous and ecologically relevant indication of the effects of exposure to chemicals remains controversial. Based on the present study, it is suggested that the evaluation of reproductive performance should never be ignored, especially in regard to ecological relevance. In comparison with other endpoints or biomarkers, reproductive performance is a more holistic parameter (Brian et al., 2007). Reproduction is dependent upon the appropriate coordination of the entire hypothalamo-pituitary-gonadal axis and therefore chemicals acting at any level of this axis via any mechanism can have adverse effects on reproduction (Brian et al., 2007). Therefore, this endpoint definitely reflects the more integrative and complex nature of biological processes compared with VTG induction or other parameters. In addition, for analyzing reproductive performance with population-level relevance (Santos et al., 2006), investigations of this endpoint are essential for understanding the population-level impacts of EDCs.

Increased attention is currently being paid to pharmaceutical substances as a class of environmental contaminants (HallingSorensen et al., 1998; Fent et al., 2006). Like other pharmaceuticals, TAM and LET can enter the aquatic environment in their native form or as transformed excretory products primarily in municipal sewage effluents, and cause ecotoxicological effects (Hilton and Thomas, 2003; Ashton et al., 2004). In a study of the environmental occurrence of TAM in the UK, the concentrations ranged from 0.146 to $0.369 \mu \mathrm{g} / \mathrm{L}$ in sewage effluents and the parent compound was not degraded in sewage treatment plants (Fent et al., 2006). Estuarine waters were found to contain concentrations ranging from 0.027 to $0.212 \mu \mathrm{g} / \mathrm{L}$ (Fent et al., 2006). Unfortunately, however, there are no currently available data for environmental concentrations of LET. Besides the two chemicals tested here and other related pharmaceuticals, a large number of chemicals present in the environment have been found to be capable of eliciting directacting antiestrogenic activity and/or inhibiting aromatase activity (Seralini and Moslemi, 2001; Ankley et al., 2002; Hinfray et al., 2006). It should be noted that the concentrations of estrogen and antiestrogens used in this laboratory study, as well as their ratios, cannot exactly reflect the real exposure situations of aquatic organisms that would be expected in environmental samples. Therefore, the present results should be interpreted with caution, and further investigations, which may be extended to chemicals at environmentally relevant concentrations with more properties (such as androgen agonists and antagonists), are required. However, our observations contribute to the current understanding of the combined effects of multicomponent mixtures of EDCs with different modes of action, and are therefore of considerable relevance to assessments of environmental risk.

\section{Conclusions}

In the present study, the combined effects of EDCs with estrogen agonist and antagonist activities were assessed using paired Japanese medaka. Natural estrogen E2 and the pharmaceutical substances TAM and LET, which are both model antiestrogens, were tested as reference compounds individually and in combination. The results revealed that even if the antiestrogens can neutralize the effects of estrogen to some extent in some biomarkers (such as VTG), the impairment in reproductive performance was hardly ameliorated or even became more severe. The data illustrate the importance of considering multiple endpoints at different biological levels of organisms for accurate and adequate assessment of the integrated effects of EDCs, especially in environmentally relevant exposure situations. Since reproductive performance is of population-level relevance, investigation of this endpoint is essential for adequate protection of wildlife against the effects of complex mixtures of EDCs.

\section{Acknowledgements}

We gratefully acknowledge the supports of National Basic Research Program of China (2007CB407304) and Natural Science Foundation of China $(20737003,20621703)$ for conducting this research.

\section{References}

Ankley, G.T., Kahl, M.D., Jensen, K.M., Hornung, M.W., Korte, J.J., Makynen, E.A., Leino, R.L., 2002. Evaluation of the aromatase inhibitor fadrozole in a short-term reproduction assay with the fathead minnow (Pimephales promelas). Toxicol. Sci. 67, $121-130$.

Ashton, D., Hilton, M., Thomas, K.V., 2004. Investigating the environmental transport of human pharmaceuticals to streams in the United Kingdom. Sci. Total Environ. 333, 167-184

Brian, J.V., Harris, C.A., Scholze, M., Kortenkamp, A., Booy, P., Lamoree, M., Pojana G., Jonkers, N., Marcomini, A., Sumpter, J.P., 2007. Evidence of estrogenic mixture effects on the reproductive performance of fish. Environ. Sci. Technol. 41 337-344.

Brion, F., Nilsen, B.M., Eidem, J.K., Goksoyr, A., Porcher, J.M., 2002. Development and validation of an enzyme-linked immunosorbent assay to measure vitellogenin in the zebrafish (Danio rerio). Environ. Toxicol. Chem. 21, 1699-1708.

Carlson, R.W., 1997. Scientific review of tamoxifen: overview from a medical oncologist. Semin. Oncol. 24, 151-157.

Ciereszko, A., Wolfe, T.D., Dabrowski, K., 2005. Analysis of DNA damage in sea lamprey (Petromyzon marinus) spermatozoa by UV, hydrogen peroxide, and the toxicant bisazir. Aquat. Toxicol. 73, 128-138.

Elias, E.E., Kalombo, E., Mercurio, S.D., 2007. Tamoxifen protects against $17 \alpha-$ ethynylestradiol induced liver damage and the development of urogenital papillae in the rainbow darter (Etheostoma caeruleum). Environ. Toxicol. Chem. 26, 1879-1889.

Fent, K., Weston, A.A., Caminada, D., 2006. Ecotoxicology of human pharmaceuticals. Aquat. Toxicol. 76, 122-159.

Filby, A.L., Neuparth, T., Thorpe, K.L., Owen, R., Galloway, T.S., Tyler, C.R., 2007. Health impacts of estrogens in the environment, considering complex mixture effects. Environ. Health Perspect. 115, 1704-1710.

Halling-Sorensen, B., Nors Nielsen, S., Lanzky, P.F., Ingerslev, F., Holten Lutzhoft, H.C. Jorgensen, S.E., 1998. Occurrence, fate and effects of pharmaceutical substances in the environment-a review. Chemosphere 36, 357-393.

Haynes, B.P., Dowsett, M., Miller, W.R., Dixon, J.M., Bhatnagar, A.S., 2003. The pharmacology of letrozole. J. Steroid Biochem. 87, 35-45.

Hilton, M.J., Thomas, K.V., 2003. Determination of selected human pharmaceutical compounds in effluent and surface water samples by high-performance liquid chromatography-electrospray tandem mass spectrometry. J. Chromatogr. A $1015,129-141$.

Hinfray, N., Porcher, J.-M., Brion, F., 2006. Inhibition of rainbow trout (Oncorhynchus mykiss) P450 aromatase activities in brain and ovarian microsomes by various environmental substances. Comp. Biochem. Physiol. C 144, 252-262.

Jobling, S., Beresford, N., Nolan, M., Rodgers-Gray, T.P., Tyler, C.R., Sumpter, J.P., 2002. Altered sexual maturation and gamete production in wild roach (Rutilus rutilus) living in rivers that receive treated sewage effluents. Biol. Reprod. 66, 272-281.

Jordan, V.C., Dix, C.J., Rowsby, L., Prestwich, G., 1977. Studies on the mechanism of action of the nonsteroidal antioestrogen tamoxifen (I.C.I. 46,474) in the rat. Mol Cell. Endocrinol. 7, 177-192.

Kang, I.J., Yokota, H., Oshima, Y., Tsuruda, Y., Yamaguchi, T., Maeda, M., Imada, N., Tadokoro, H., Honjo, T., 2002. Effect of $17 \beta$-estradiol on the reproduction of Japanese medaka (Oryzias latipes). Chemosphere 47, 71-80.

Kawahara, T., Yamashita, I., 2000. Estrogen-independent ovary formation in the medaka fish, Oryzias latipes. Zool. Sci. 17, 65-68.

Kortenkamp, A., 2007. Ten years of mixing cocktails: a review of combination effects of endocrine-disrupting chemicals. Environ. Health Perspect. 115, 98-105.

Martinovi, D., Blake, L.S., Durhan, E.J., Greene, K.J., Kahl, M.D., Jensen, K.M., Makynen, E.A., Villeneuve, D.L., Ankley, G.T., 2008. Reproductive toxicity of vinclozolin in the fathead minnow: confirming an anti-androgenic mode of action. Environ. Toxicol. Chem. 27, 478-488.

Mihailescu, R., Aboul-Enein, H.Y., Efstatide, M.D., 2000. Identification of tamoxifen and metabolites in human male urine by GC/MS. Biomed. Chromatogr. 14 180-183.

Nimrod, A.C., Benson, W.H., 1998. Reproduction and development of Japanese medaka following an early life stage exposure to xenoestrogens. Aquat. Toxicol. 44, 141-156. 
Packard, G.C., Boardman, T.J., 1999. The use of percentages and size-specific indices to normalize physiological data for variation in body size: wasted time, wasted effort? Comp. Biochem. Physiol. A 122, 37-44.

Patyna, P.J., Davi, R.A., Parkerton, T.F., Brown, R.P., Cooper, K.R., 1999. A proposed multigeneration protocol for Japanese medaka (Oryzias latipes) to evaluate effects of endocrine disruptors. Sci. Total Environ. 233, 211-220.

Purdom, C., Hardiman, P., Bye, V., Eno, N., Tyler, C., Sumpter, J., 1994. Estrogenic effects of effluents from sewage treatment works. Chem. Ecol. 8, 275-285.

Pfister, C.U., Duval, M., Godbillon, J., Gosset, G., Gygax, D., Marfil, F., Sioufi, A., Winkler, B., 1994. Development, application and comparison of an enzyme immunoassay and a high-performance liquid chromatography method for the determination of the aromatase inhibitor CGS 20267 in biological fluids. J. Pharmacol. Sci. 83, 520-524.

Quintana, J.B., Carpinteiro, J., Rodríguez, I., Lorenzo, R.A., Carro, A.M., Cela, R., 2004. Determination of natural and synthetic estrogens in water by gas chromatography with mass spectrometric detection. J. Chromatogr. A. 1024, 177-185.

Rodriguez-Mozaz, S., Marco, M.-P., Lopez de Alda, Maria, J., Barceló, D., 2004. Biosensors for environmental monitoring of endocrine disruptors: a review article. Anal. Bioanal. Chem. 378, 588-598.

Santos, M.M., Micael, J., Carvalho, A.P., Morabito, R., Booy, P., Massanisso, P., Lamoree, M., Reis-Henriques, M.A., 2006. Estrogens counteract the masculinizing effect of tributyltin in zebrafish. Comp. Biochem. Physiol. C 142, 151-155.

Seralini, G.-E., Moslemi, S., 2001. Aromatase inhibitors: past, present and future. Mol. Cell. Endocrinol. 178, 117-131.

Sheahan, D.A., Brighty, G.C., Daniel, M., Kirby, S.J., Hurst, M.R., Kennedy, J., Morris, S. Routledge, E.J., Sumpter, J.P., Waldock, M.J., 2002. Estrogenic activity measured in a sewage treatment works treating industrial inputs containing high concen- trations of alkylphenolic compounds-a case study. Environ. Toxicol. Chem. 21 507-514.

Smith, I.E., 1999. Aromatase inhibitors: a dose-response effect? Endocr. Rel. Cancer 6, 245-249.

Sun, L., Zha, J., Spear, P.A., Wang, Z., 2007a. Tamoxifen effects on the early life stages and reproduction of Japanese medaka (Oryzias latipes). Environ. Toxicol. Pharmacol. 24, 23-29.

Sun, L., Zha, J., Spear, P.A., Wang, Z., 2007b. Toxicity of the aromatase inhibitor letrozole to Japanese medaka (Oryzias latipes) eggs, larvae and breeding adults. Comp. Biochem. Physiol. C 145, 533-541.1.

Thorpe, K.L., Gross-Sorokin, M., Johnson, I., Brighty, G., Tyler, C.R., 2006. An assessment of the model of concentration addition for predicting the estrogenic activity of chemical mixtures in wastewater treatment works effluents. Environ. Health Perspect. 114, 90-97.

Tyler, C.R., Jobling, S.R., Sumpter, J.P., 1998. Endocrine disruption in wildlife: a critical review of the evidence. Crit. Rev. Toxicol. 28, 319-361.

U.S.EPA, 2002. Draft detailed review paper on fish screening assays for endocrine disruption. Columbus, Ohio.

Van der Ven, L.T.M., Van den Brandhof, E.-J., Vos, J.H., Wester, P.W., 2007. Effects of the estrogen agonist $17 \beta$-estradiol and antagonist tamoxifen in a partial life-cycle assay with zebrafish (Danio rerio). Environ. Toxicol. Chem. 26, 92-99.

Witorsch, R.J., 2002. Endocrine disruptors: can biological effects and environmental risks be predicted? Regul. Toxicol. Pharmacol. 36, 118-130.

Yamamoto, T., 1953. Artificially induced sex-reversal in genotypic males of the medaka (Oryzias latipes). J. Exp. Zool. 123, 571-594.

Yamanaka, S., Arizono, K., Matsuda, Y., 1998. Development and application of an effective detection method for fish plasma vitellogenin induced by environmental estrogens. Biosci. Biotechnol. Biochem. 62, 1196-1200. 